\title{
A Dorsal CA2 to Ventral CA1 Circuit Contributes to Oxytocinergic Modulation of Long-Term Social Recognition Memory
}

\section{Tsung-Chih Tsai}

National Cheng Kung University College of Medicine

\section{Yi-Syuan Fang}

National Cheng Kung University College of Medicine

\section{Yu-Chieh Hung}

National Cheng Kung University College of Medicine

\section{Ling-Chien Hung}

Ditmanson Medical Foundation Chia-Yi Christian Hospital

Kuei-Sen Hsu ( $\nabla$ richard@mail.ncku.edu.tw)

National Cheng Kung University https://orcid.org/0000-0002-3498-5157

\section{Research Article}

Keywords: Oxytocin, Oxytocin receptor, Social recognition memory, CA2, Hippocampus, Paraventricular nucleus

Posted Date: February 18th, 2022

DOI: https://doi.org/10.21203/rs.3.rs-1356484/v1

License: (c) (1) This work is licensed under a Creative Commons Attribution 4.0 International License. Read Full License 


\section{Abstract}

Social recognition memory (SRM) is the ability to distinguish familiar from novel conspecifics and is crucial for survival and reproductive success across social species. We previously reported that oxytocin (OXT) receptor (OXTR) signaling in the CA2/CA3a of dorsal hippocampus is essential to promote the persistence of long-term SRM, yet how the endogenous OXT system influences CA2 outputs to regulate long-term SRM formation remains unclear. Here, using a transgenic mouse line (Amigo2-Cre) that expresses the Cre recombinase predominantly in CA2 pyramidal neurons, we show that selective ablation of Oxtrin the CA2 $\left(\mathrm{Oxtr}^{-/-}\right)$suffices to impair the persistence of long-term SRM but has no effect on sociability and social novelty preference in the three-chamber paradigm test. We find that cell-type specific activation of OXT neurons within the hypothalamic paraventricular nucleus enhances long-term SRM and this enhancement is blocked by local application of OXTR antagonist L-368,899 into dorsal hippocampal CA2 (dCA2) region. In addition, chemogenetic neuronal silencing in dCA2 demonstrated that neuronal activity is essential for forming long-term SRM. Moreover, chemogenetic terminal-specific inactivation revealed a crucial role for dCA2 outputs to ventral CA1 (vCA1), but not dorsal lateral septum, in long-term SRM. Finally, targeted activation of the dCA2-to-vCA1 circuit effectively ameliorated longterm SRM deficit observed in $\mathrm{Oxtr}^{-/-}$mice. Together, these findings highlight the importance of hippocampal CA2 OXTR signaling in governing the persistence of long-term SRM and identify a hippocampal circuit linking dCA2 to vCA1 necessary for controlling long-term SRM formation.

\section{Introduction}

Social recognition memory (SRM) refers to the ability of animals and humans to recognize and memorize familiar conspecifics and this ability is critical for maintaining appropriate social relationships [1-3]. In animals, SRM is essential for forming social groups and establishing hierarchies and social and affective ties $[4,5]$. The development of SRM in humans and primates relies heavily on the use of visual and auditory cues, whereas other mammals such as rodents rely their SRM processing on multisensory integration of olfactory, auditory and somatosensory cues $[4,6]$. Previous studies on the neural basis of SRM formation in mice have implicated multiple brain regions, including the hippocampus, amygdala and anterior cingulate cortex [7-13]. The hippocampus in particular has been firmly established as a key region in SRM formation. Indeed, bilateral lesions of the hippocampus with ibotenic acid result in an impaired SRM in mice [7]. Thus, the hippocampus is an attractive brain region for the study of neurobiological mechanisms underlying SRM. Within the hippocampus, distinct subregions may contribute differentially to encode and retrieve mnemonic information through their differential connections. Of these subregions, recent studies have shed light on the importance of dorsal CA2 (dCA2) in SRM processing. For example, excitatory lesion or genetically targeted inactivation of dCA2 pyramidal neurons was found to selectively impair SRM with no change in sociability $[9,13,14]$. Additional studies have shown that dCA2 contributes to SRM by providing strong excitatory inputs to ventral CA1 (vCA1) that contains a particular subset of neurons implicated in SRM storage $[13,15]$. Furthermore, by interfering with different phases of social memory tasks, Meira et al. [13] showed that dCA2 is necessary 
for all phases of SRM, including encoding, consolidation and recall. Despite these observations, much less is known about the neuromodulatory mechanisms engaged CA2 circuits in regulating SRM formation.

The hypothalamic neuropeptide oxytocin (OXT) promotes various aspects of social behaviors [16, 17]. OXT is mainly synthesized in neurons of the paraventricular nucleus (PVN) and supraoptic nucleus. While the vast majority of OXT neurons project to the posterior pituitary, they also sent axon collaterals to various forebrain regions that express OXT receptors (OXTRs), including the central nucleus of the amygdala, lateral septum (LS), nucleus accumbens, medial prefrontal cortex and hippocampus [18-20]. We have recently shown that OXTRs are prominently expressed in hippocampal CA2 and CA3 pyramidal neurons of mice and conditional deletion of CA2/CA3a Oxtr impairs the persistence of long-term SRM [12]. Accordingly, viral-mediated deletion of Oxtrin anterior CA2/CA3 neurons has been shown to impair discrimination of social, but not non-social, stimuli in mice [21]. Nonetheless, silencing dCA3 alone had no significant effect on SRM [22]. These findings highlight the importance of CA2 OXTR signaling in modulating the persistence of SRM. Nevertheless, it remains clear how the endogenous OXT system and CA2 outputs regulate long-term SRM formation. Here, to specifically examine the functional and behavioral relevance of CA2 OXTRs, we used a CA2-selective Amigo2-Cre mouse line to obtain selective deletion of Oxtr in CA2 neurons [9]. To bridge of our understanding of endogenous OXTergic neuromodulation, we determined the impact of selective chemogenetic activation of PVN OXT neurons on long-term SRM formation. Finally, we explored CA2 outputs that underpin long-term SRM formation using circuit-based chemogenetic approaches. We report that the endogenous OXT system strongly promotes the persistence of long-term SRM by acting through the dCA2-to-vCA1 circuit.

\section{Materials And Methods}

\section{Animals}

All experimental procedures were approved by the Institutional Animal Care and Use Committee (IACUC) at the National Cheng Kung University (authorization \#107023) and conformed to the National Institutes of Health Guide for the Care and Use of Laboratory Animals. Homozygous Oxtr-floxed $(\mathrm{Oxtr} / \mathrm{J}$; donating investigator: Dr. W. Scott Young III) and heterozygous Amigo2-Cre transgenic mice (donating investigator: Dr. Steven A. Siegelbaum) were obtained from The Jackson Laboratory. We crossed Amigo2-Cre mice with $O x t r / J$ mice to generate CA2-specific Oxtrconditional knockout $\left(\mathrm{Oxtr}^{-/-}\right)$mice, which are maintained on the C57BL/ 6 genetic background. The heterozygous OXTR-Venus knock-in (Oxtr $\left.{ }^{\text {Venus-Neo/+ }}\right)$ mice were obtained from Dr. Katsuhiko Nishimori and characterized as described previously [23]. Genotyping was performed by the polymerase chain reaction (PCR)-based method using genomic DNA isolated from tail samples as previously described [24]. All mice were housed in groups of three with same-sex littermates, kept in a $12 \mathrm{~h}$ light/12 $\mathrm{h}$ dark cycle and had ad libitum access to food and water, in the humidity- and temperature-controlled $\left(25 \pm 1^{\circ} \mathrm{C}\right)$ specific pathogen-free animal facility. Experiments were done with 
adult male mice starting at 10-12 weeks old and all behavioral testing were performed in the light phase. All experiments were conducted blinded to the genotype and experimental designs.

\section{Recombinant adeno-associated virus (AAV) vector production}

DNA plasmids encoding pAAV-hSyn-DIO-mCherry (Addgene, plasmid \#50459), pAAV-hSyn-DIO-hM3D(Gq)mCherry (Addgene, plasmid \#44361), pAAV-hSyn-DIO-hM4D(Gi)-mCherry (Addgene, plasmid \#44362) and pAAV-m0XT-hM3D(Gq)-mCherry (Addgene, plasmid \#70717) were purchased from Addgene. Plasmid DNA was amplified, purified and collected with the QIAGEN plasmid maxiprep kit following manufacturer's instructions. The purified plasmids were mixed into $\mathrm{CaCl}_{2}$ solution with the DNA plasmid coding AAV5 and co-transfected into HEK293GP cells using the calcium phosphate co-precipitation method as described previously $[12,24]$. Transfected cells were harvested $72 \mathrm{~h}$ after transfection and viruses were purified using the AAV Purification Mega Kit (Cell Biolabs, Inc.). Viral titers were $5 \cdot 10^{12}$ particles $/ \mathrm{ml}$ and stored in aliquots at $-80^{\circ} \mathrm{C}$ until use.

\section{Stereotaxic viral injections and chemogenetic manipulations}

Under isoflurane ( $5 \%$ induction and $2 \%$ maintenance; Attane) anesthesia, concentrated virus-stock solution was stereotaxically injected into the targeted sites $(0.5 \mu \mathrm{l}$ per site at $0.25 \mu \mathrm{l} / \mathrm{min})$ by using a Hamilton syringe with a 34-gauge blunted-tip needle. Buprenorphine $(0.1 \mathrm{mg} / \mathrm{kg})$ was subcutaneously delivered prior to the surgery to reduce discomfort. Body temperature was maintained at $37^{\circ} \mathrm{C}$ via a heating pad. For chemogenetic activation of PVN OXT neurons, we bilaterally injected $\mathrm{AAV}_{5}-\mathrm{mOXT}-$ hM3D $(\mathrm{Gq})$-mCherry into the PVN of the hypothalamus according to the following injection coordinates: anteroposterior (AP) $-0.8 \mathrm{~mm}$, mediolateral $(\mathrm{ML}) \pm 0.2 \mathrm{~mm}$ and dorsoventral (DV) $-5 \mathrm{~mm}$. For chemogenetic manipulation of dCA2, we bilaterally injected AAV ${ }_{5}$-hSyn-DIO-mCherry, AAV ${ }_{5}$-hSyn-DIOhM4D(Gi)-mCherry or AAV 5 -hSyn-DIO-hM3D(Gq)-mCherry into the hippocampus of Amigo2-Cre or Oxtr-/mice according to the following injection coordinates: AP $-2.3 \mathrm{~mm}, \mathrm{ML} \pm 2.7 \mathrm{~mm}$ and DV $-2.0 \mathrm{~mm}$. For silencing of dCA2-to-vCA1 projections, $\mathrm{AAV}_{5}$-hSyn-DIO-hM4D(Gi)-mCherry was bilaterally injected into dCA2 and mice were bilaterally implanted with 26 gauge cannula guides (RWD Life Science Co., Ltd.) aimed at vCA1 (AP -3.0 mm, ML $\pm 2.5 \mathrm{~mm}$ and DV -2.0 mm) to deliver vehicle (PBS, $0.5 \mu \mathrm{l}$ ) or clozapine-Noxide (CNO, 4 mM in PBS, $0.5 \mu$; Sigma-Aldrich). For silencing of dCA2-to-dorsal LS (dLS) projections, $A A V_{5}$-hSyn-DIO-hM4D(Gi)-mCherry was bilaterally injected into dCA2 and mice were bilaterally implanted with 26 gauge cannula guides aimed at dLS (AP $+0.2 \mathrm{~mm}, \mathrm{ML} \pm 0.4 \mathrm{~mm}$ and DV $-2.5 \mathrm{~mm}$ ) to deliver vehicle (PBS, $0.5 \mu \mathrm{l}$ ) or CNO (4 mM in PBS, $0.5 \mu \mathrm{l})$. Dummy cannulas were inserted into guide cannulas and secured to skull with dental cement. All injections were performed on 10-12 weeks old male mice and were followed by a 3-week viral incubation period before the start of the behavioral testing. For chemogenetic activation of PVN OXT projection to dCA2, CNO (4 mM in PBS, $0.5 \mu$ l) was delivered locally through cannulas implanted bilaterally into dCA2 20 min before performing the three-chamber paradigm test. To better characterize the involvement of locally released OXT and subsequent activation of OXTR, 
L-368,899 (12 $\mu \mathrm{g}$ in PBS, $0.5 \mu \mathrm{l}$, Tocris Bioscience) was administrated bilaterally into the dCA2 $10 \mathrm{~min}$ before CNO injection. Drug dose of L-368,899 was selected on the basis of published study [21]. For chemogenetic silencing of dCA2 neurons, Amigo2-Cre mice infected in dCA2 with AAV 5 -hSyn-DIO$\mathrm{hM} 4 \mathrm{D}(\mathrm{Gi})-\mathrm{mCherry}$ or control $\mathrm{AAV}_{5}$-hSyn-DIO-mCherry were injected intraperitoneally with vehicle $(5 \%$ DMSO in PBS) or CNO (5 mg/kg in 5\% DMSO) 30 min before performing the three-chamber paradigm test. Dose of CNO was selected on the basis of published studies $[25,26]$. After behavioral testing, brains were dissected and serial slices were imaged to verify correct viral expression and implant placement location.

\section{Fluorescent in situ hybridization}

Fluorescent in situ hybridization (FISH) analysis was performed using RNAscope® Multiplex Fluorescent Reagent Kit 2.0 manufacturer's instructions (Advanced Cell Diagnostics; RRID: SCR_012481) as previously described $[12,24]$. Coronal brain slices $(16 \mu \mathrm{m})$ were fixed in $4 \%$ paraformaldehyde (PFA; Sigma-Aldrich) for $15 \mathrm{~min}$ and dehydrated in a 50\%, 70\% and 100\% ethanol series for 5 min each. Sections were subjected to reagent Pretreat 3 at $25^{\circ} \mathrm{C}$ for $30 \mathrm{~min}$ and then hybridized with probes at $40^{\circ} \mathrm{C}$ for $2 \mathrm{~h}$ in a humidified oven (ACD HybEZ ${ }^{\text {TM }}$ Hybridization System). The Oxtr-01 probe (Cat\# 454011) and the Amigo2 probe (Cat\# 504478) were used to target Oxtr mRNA and Amigo2 mRNA, respectively. After hybridization, brain sections were sequentially applied with a series of probe signal amplification steps, washed with ACD Wash Buffer 2 times, 2 min each wash, and then mounted with VECTASHIELD antifade mounting medium (Vector Laboratories) containing 4',6-diamidino-2-phenylindole (DAPI, 1:5000; Cat\# D9542; Sigma-Aldrich) for staining DNA.

\section{Histology and quantification}

Tissue preparation and histological analysis was performed as described previously [12]. Mice were deeply anesthetized with $5 \%$ isoflurane and perfused transcardially with cold $\left(4^{\circ} \mathrm{C}\right) \mathrm{PBS}$, followed by $4 \%$ PFA in 0.1 M PBS, pH 7.4. After the perfusion, brains were removed and fixed in $4 \%$ PFA for $24 \mathrm{~h}$ at $4^{\circ} \mathrm{C}$ and then immersed for at least $48 \mathrm{~h}$ in the solution containing $30 \%$ sucrose at $4^{\circ} \mathrm{C}$ before sectioning. Coronal brain slices containing the hippocampus were sectioned ( $20 \mu \mathrm{m}$ thickness), washed with $0.3 \%$ Triton X-100, and then incubated with blocking solution containing 3\% goat serum in PBS. For quantitative analysis of neuron numbers with Nissl staining, sections were mounted directly on gelatincoated glass slides and dried. The slides were stained with $1.0 \%$ cresyl violet, dehydrated through a series of ethanol, cleared and coverslipped with Permount (Thermo Fisher Scientific). Nissl staining within the CA2 region was quantified in images from about 1.5 to $2.5 \mathrm{~mm}$ posterior to Bregma every sixth coronal section captured at 200x magnification and digitized with an Olympus BX51 microscope equipped with Olympus DP70 digital camera. All images were imported into NIH ImageJ software (RRID:SCR_001935) for analysis.

\section{Immunohistochemistry}

Immunofluorescence staining was performed as described previously [12, 24]. In brief, Oxtr ${ }^{\mathrm{Venus}-\mathrm{Neo} /+}$ mice were deeply anesthetized with $5 \%$ isoflurane and perfused transcardially with $4^{\circ} \mathrm{C}$ PBS, followed by 
4\% PFA in 0.1 M PBS, pH 7.4. After the perfusion, brains were removed and fixed in $4 \%$ PFA for $24 \mathrm{~h}$ at $4^{\circ} \mathrm{C}$ and then transferred to the solution containing $30 \%$ sucrose that immersed in $4^{\circ} \mathrm{C}$ for at least $48 \mathrm{~h}$ before slicing. Coronal slices were sectioned to a $40 \mu \mathrm{m}$ thickness, washed with 0 . \% Triton X-100, and then incubated for blocking with solution containing 3\% goat serum in PBS for $30 \mathrm{~min}$. After blocking, the sections were incubated with primary antibody against striatum-enriched protein-tyrosine phosphatase (STEP; also known as PTPN5) (1:500, Cell Signaling Technology, Cat\#4376; RRID: AB_1904101). Finally, sections were washed with TBS-T (10 mM Tris-HCl, $150 \mathrm{mM} \mathrm{NaCl}$ and $0.025 \%$ Tween 20; $\mathrm{pH} 7.4)$ and then incubated with Alexa Fluor 568 antibody (1:500, Life Technologies) secondary antibody for $2 \mathrm{~h}$ at room temperature in blocking solution. The immunostained sections were collected on separate gelatinsubbed glass slides, rinsed extensively in PBS, and mounted with ProLong Gold Antifade Reagent (Invitrogen). Post hoc tissue images were acquired using an Olympus FluoView FV3000 confocal laser scanning microscope. All images were imported into $\mathrm{NIH}$ ImageJ software for analysis.

\section{Three-chamber paradigm test}

The three-chamber paradigm test was conducted as previously described [12]. The apparatus was a custom-built rectangular box $(60 \mathrm{~cm} \cdot 40 \mathrm{~cm} \cdot 22 \mathrm{~cm})$ divided into three chambers made from clear polycarbonate, with openings ( $10 \mathrm{~cm}$ width $\times 5 \mathrm{~cm}$ height) that allow access into each chamber. The assay consisted of three trials in a dimly lit room (10-15 lux white light). The subject mouse was initially placed into the box and allowed to freely explore all three chambers for $10 \mathrm{~min}$ for habituation. After habituation phase, a juvenile male mouse (stimulus, $3-5$ weeks old), which had no previous contact with the subject mice, was placed in a wire cage of left or right chamber (systemically alternated) and an empty wire cage was placed in the other side chamber. The subject mouse was placed in the middle chamber, and then the mouse was allowed to freely explore all three chambers for 5 min (sociability test). The time that the test subject spent in sniffing at the wire cage containing the juvenile stimulus mouse or the empty wire cage was measured. At the end of the sociability test, the subject mouse was gently guided to the center chamber while the empty wire cage was replaced with a novel unfamiliar male juvenile mouse (novel 1). The subject mouse again freely explored all three chambers for $5 \mathrm{~min}$ to quantify social preference for a novel stranger mouse (social novelty preference test). The subject mouse had a free choice between the first, already-investigated mouse (familiar) and the novel unfamiliar mouse. The time that the test subject spent in sniffing at the wire cage containing a familiar mouse or a novel mouse was measured. After social novelty test, mice were returned to their home cages. On day 2 or day 8, 1-day or 7-day long-term SRM was examined in which the familiar mouse was placed in the left or right chamber, and a novel unfamiliar male juvenile mouse (novel 2) was placed in the cage of other chamber. The subject mouse again freely explored all three chambers for $5 \mathrm{~min}$. The subject mouse had a free choice between the familiar mouse and the novel unfamiliar mouse for $5 \mathrm{~min}$. The time that the test subject spent in sniffing at the wire cage containing a familiar mouse or a novel mouse was measured. The behavior of the animals was videotaped and analyzed using the EthoVision XT video tracking system (Noldus; RRID: SCR_000441). The discrimination index for assessing sociability was calculated as [(time of sniffing the stimulus object - time of sniffing the empty cage)/ (time of sniffing in both stimulus object and empty cage)]. The discrimination index for assessing social memory was calculated 
as [(time of sniffing the novel object - time of sniffing the familiar object)/ (time of sniffing the objects in both novel and familiar object)].

\section{Statistical analysis}

No statistical methods were used to predetermine sample size, but the number of animals used in each experiment was chosen on the basis of previous studies in our laboratory [12,24]. No specific randomization method was used in allocating an animal into a particular experimental conditions reported in this study. The data were expressed as mean \pm SEM and statistical comparisons were conducted using Prism 6 software (GraphPad). Two-tailed paired Student's $t$-test was used for withingroup comparison and the unpaired Student's $t$-test was used for comparison between two independent groups. Number of animals used is indicated by $n$. Probability values of $p<0.05$ were considered to represent significant differences.

\section{Results}

\section{Conditional deletion of Oxtr from CA2 excitatory neurons}

To first ensure the expression of OXTRs in hippocampal dCA2 subregion, we performed immunohistochemical staining using a transgenic $\mathrm{Oxtr}^{\mathrm{V} \text { enus-Neo/+ }}$ mouse line that expresses the Venus variant of yellow fluorescent protein in OXTR-expressing cells. Consistent with previous reports [23, 24], we observed strong expression of Venus-labeled cells in dCA2 stratum pyramidale of Oxtr ${ }^{\text {Venus-Neo/+ }}$ mice. As illustrated by a single atlas plate schmetic (bregna $-2.3 \mathrm{~mm}$ ), double-labeling revealed colocalized expression of OXTR and putative CA2 marker STEP (Fig. 1A), indicating that OXTRs are indeed expressed in CA2 pyramidal neurons $[27,28]$. To gain insight into the functional and behavioral relevance of OXTRs in CA2 pyramidal neurons, we took advantage of the Cre-loxP recombination approach to conditionally delete Oxtrfrom CA2 excitatory neurons by crossing $O x t r^{f / f}$ mice with Amigo2-Cre mice, in which Cre expression is largely restricted to CA2 pyramidal neurons [9, 29]. PCR screening of mouse genomic tail DNA confirmed heterozygous $\left(\mathrm{Oxtr}^{+/-}\right)$and homozygous $\mathrm{Oxtr}\left(\mathrm{Oxtr}^{-1-}\right)$ conditional knockout mice (Fig. 1B). Consistently, a dual-probe FISH revealed that the majority of Oxtr mRNA-positive cells were Amigo 2 mRNA-expressing cells and the number of Oxtr-positive cells in the CA2 of Oxtr ${ }^{-1-}$ mice was markedly reduced compared to that of WT $\left(\mathrm{Oxtr}^{\mathrm{f} / \mathrm{f}}\right)$ mice (Fig. 1C), confirming the efficiency of Cre-loxPmediated deletion of CA2 Oxtr. However, histological analysis by cresyl violet staining revealed that Oxtr deletion did not significantly affect the total number of neurons in CA2 stratum pyramidale compared to WT littermates (WT: $n=3 ;$ Oxtr $^{-1-}: n=4 ; t_{(5)}=0.19, p=0.85$; unpaired Student's $t$-test; Fig. 1D).

\section{Conditional deletion of CA2 Oxtr impairs the persistence of long-term SRM}

To determine the impact of CA2 OXTR deletion on SRM persistence, we used a three-chamber paradigm, a task widely used for studying social approach behaviors in mice $[7,30]$. We initially compared the performance of WT and $\mathrm{Oxtr}^{-/-}$mice in sociability assay, which examines the subject mouse's preference 
for interacting with a social stimulus (a unfamiliar mouse enclosed in a wire cage) over a non-social one (an empty wire cage). We found no difference between WT and Oxtr ${ }^{-1-}$ mice in preference for the wire cage containing the stimulus mouse. Discrimination index was similar between WT $(n=16)$ and $O x t r^{-/-}$ mice $(n=16)$ in sociability test $\left(t_{(30)}=1.78, p=0.09\right.$; unpaired Student's $t$-test; Fig. 2A). We next performed the social novelty preference test that examines the ability of the subject mouse to discriminate between novel and familiar social stimuli. Following the sociability test, a novel stimulus mouse was presented in the previously empty wire cage. As shown in Fig. 2B, both WT $(n=16)$ and Oxtr ${ }^{-/-}$mice $(n=16)$ exhibited a more extensive investigation of the novel mouse compared to the familiar mouse, with no difference between the genotypes. Discrimination index was comparable between WT and $\mathrm{Oxtr}^{-1-}$ mice in social novelty preference test $\left(t_{(30)}=1.18, p=0.25\right.$; unpaired Student's $t$-test). When mice were tested SRM one day after the training session (1-day long-term SRM), both WT $(n=8)$ and $O x t^{-1-}$ mice $(n=8)$ revealed intact memory retention. They both displayed a significant and similar preference for spending more time in exploring the novel mouse than the familiar mouse. There was no significant differences between WT and $\mathrm{Oxtr}^{-/-}$mice in discrimination index of 1-day long-term SRM test $\left(t_{(14)}=0.45, p=0.66\right.$; unpaired Student's $t$-test; Fig. 2C). In contrast, when tested SRM 7 days after the initial interaction (7-day long-term SRM), we found that $\mathrm{Oxtr}^{-1-}$ mice $(n=8)$ were unable to discriminate between novel and familiar mouse as they spent equal time investigating both the novel and familiar mouse $\left(t_{(7)}=0.45, p=0.66\right.$; paired Student's $t$-test), indicating the degree of 7-day long-term SRM was impaired by Oxtr deletion. WT mice ( $n$ $=8)$, however, spent significantly more time exploring the novel mouse than the familiar mouse $\left(t_{(7)}=4.05\right.$ $p=0.005$; paired Student's $t$-test). As a consequence, a statistically significant discrimination index was observed between WT and $O x t r^{-1-}$ mice in 7-day long-term SRM $\left(t_{(14)}=2.27, p=0.039\right.$; unpaired Student's $t$-test; Fig. 2D). Together, these results suggest that CA2 OXTRs is crucial for the persistence of long-term SRM, but not for sociability, social novelty preference and 1-day SRM.

\section{Targeted activation of PVN OXT neurons promotes the persistence of long-term SRM}

We and others previously reported that OXT neurons in the PVN send axonal projections to dCA2 [12, 28, 31]. To determine if the endogenous OXT system participates in regulating the formation of long-term SRM, we used chemogenetic approach to activate PVN OXT neurons during SRM encoding. We bilaterally injected the PVN with an $A A V_{5}$ vector expressing $h M 3 D(G q)$-mCherry under the control of the endogenous mouse OXT (mOXT) promoter [AAV ${ }_{5}$-mOXT-hM3D(Gq)-mCherry] (Fig. 3A). Three weeks after viral injection, mice were subjected to bilateral injection of either vehicle or CNO into dCA2 and then subjected to the three-chamber paradigm test $20 \mathrm{~min}$ after vehicle or $\mathrm{CNO}$ injection. We verified the neuronal specificity of viral expression by imaging mCherry, whose expression was confined to neurons coexpressing OXT in the PVN (Fig. 3A). We found that acute in vivo activation of the PVN OXT neurons with CNO $(n=12)$ did not affect sociability $\left(t_{(22)}=0.28, p=0.78\right.$; unpaired Student's $t$-test; Fig. $\left.3 \mathrm{~B}\right)$ or social novelty preference $\left(t_{(22)}=0.57, p=0.58\right.$; unpaired Student's $t$-test; Fig. $\left.3 \mathrm{C}\right)$ compared with vehicle 
treatment group $(n=12)$. Although no differences between groups were present in sociability and social novelty preference tests, acute activation of the PVN OXT neurons (hM3D $(\mathrm{Gq}) / \mathrm{CNO}$ group) caused a significant increase in the amount of 7-day long-term SRM. Discrimination index was significantly higher in the $\mathrm{hM} 3 \mathrm{D}(\mathrm{Gq}) / \mathrm{CNO}$-treated group $(n=12)$ than in the $\mathrm{hM} 3 \mathrm{D}(\mathrm{Gq}) /$ vehicle-treated group $\left(n=12 ; \mathrm{t}_{(22)}=\right.$ 3.19, $p=0.0043$; unpaired Student's $t$-test; Fig. 3D).

To better characterize the involvement of locally released OXT and subsequent activation of OXTRmediated signaling in dCA2 during SRM encoding, we bilaterally administered vehicle or an OXTR antagonist L-368,899 (12 $\mu \mathrm{g}$ in PBS, $0.5 \mu \mathrm{l}$ ) into dCA2 of hM3D(Gq)-mCherry-expressed mice $10 \mathrm{~min}$ before $\mathrm{CNO}$ injection, followed by three-chamber paradigm test $20 \mathrm{~min}$ after $\mathrm{CNO}$ injection. Post hoc histological examination of brain sections revealed robust co-expression of mCherry with OXT in the PVN (Fig. 4A). In comparison with vehicle-treated mice, we found no significant effect of L-368,899 treatment in sociability $\left(t_{(14)}=0.31, p=0.76\right.$; unpaired Student's $t$-test; Fig. 4B) or social novelty preference $\left(t_{(14)}=\right.$ $1.69, p=0.11$; unpaired Student's $t$-test; Fig. $4 \mathrm{C}$ ) in $\mathrm{hM} 3 \mathrm{D}(\mathrm{Gq}) / \mathrm{CNO}$ mice. However, pretreatment with L368,899 blocked the enhancement of 7-day long-term SRM seen in hM3D(Gq)-mCherry-expressed mice following $\mathrm{CNO}$ treatment. Post hoc analyses indicated that the enhancement of discrimination ratio seen with activation of the PVN OXT neurons was abolished when OXTRs were blocked $\left(t_{(14)}=3.43, p=0.004\right.$; unpaired Student's $t$-test; Fig. 4D). These results suggest that increasing endogenous hypothalamic OXT secretion in dCA2 can promote the persistence of long-term SRM.

\section{dCA2 activity is essential for long-term SRM formation}

To probe causality between dCA2 activity and the formation of long-term SRM, we targeted dCA2 pyramidal neurons for chemogenetic silencing during SRM encoding. We compared the effects of intraperitoneal injection of CNO in Amigo2-Cre mice infected in dCA2 with $\mathrm{AAV}_{5}$-hSyn-DIO-hM4D(Gi)mCherry or control $\mathrm{AAV}_{5}$-hSyn-DIO-mCherry. Three weeks after viral infection, all mice underwent threechamber paradigm test $30 \mathrm{~min}$ after $\mathrm{CNO}$ injection $(5 \mathrm{mg} / \mathrm{kg})$. Post hoc histological examination of brain sections revealed robust and bilateral co-expression of mCherry with STEP in dCA2 pyramidal neurons (Fig. 5A). Both groups exhibited significant preference for the wire cage containing the stimulus mouse than the empty wire cage in sociability test (Fig. 5B) and the novel mouse than the familiar mouse in social novelty preference test (Fig. $5 \mathrm{C}$ ). We found no main effects in discrimination index of sociability (mCherry + CNO: $n=8 ; \mathrm{hM} 4 \mathrm{D}(\mathrm{Gi})+\mathrm{CNO}: n=8 ; t_{(14)}=0.49, p=0.64$; unpaired Student's $t$-test; Fig. $5 \mathrm{~B}$ ) and social novelty preference (mCherry + CNO: $n=8$; $\mathrm{hM} 4 \mathrm{D}(\mathrm{Gi})+\mathrm{CNO}: n=8 ; t_{(14)}=0.42, p=0.68$; unpaired Student's $t$-test; Fig. $5 \mathrm{C}$ ) between groups. However, we observed a significant effect in 7-day long-term SRM between groups. Post hoc analysis indicated that discrimination index was significantly less for DIO-hM4D(Gi)-mCherry-expressed mice $(n=8)$ than control mCherry-expressed mice $\left(n=8 ; t_{(14)}=3.82, p=\right.$ 0.002; unpaired Student's t-test; Fig. 5D).

\section{A selective dCA2-to-vCA1 circuit involves in long-term SRM formation}


To explore dCA2 outputs necessary for controlling long-term SRM formation, we focused on vCA1 and dLS that have been identified as downstream targets of dCA2 and potential neuroanatomical substrates involved in social and emotional behaviors $[13,29]$. To interrogate the role of dCA2-to-vCA1 projections in long-term SRM, we performed bilateral injections of AAV ${ }_{5}$-hSyn-DIO-hM4D(Gi)-mCherry into dCA2 of Amigo2-Cre mice. After allowing two weeks for viral expression, mice were implanted with cannula targeting vCA1. One week later, mice underwent three-chamber paradigm test. Twenty minutes before sociability test, mice were injected with vehicle or CNO via cannula targeting vCA1. Post hoc histological examination of brain sections revealed robust co-expression of mCherry with STEP in dCA2 (Fig. 6A, bottom left panel). We also observed dense projection fibers from dCA2 to vCA1 (Fig. 6A, bottom right panel). In comparison with vehicle-treated mice $(n=7)$, we found no significant effect of CNO treatment $(n=7)$ in discrimination index of sociability $\left(t_{(12)}=1.02, p=0.33\right.$; unpaired Student's $t$-test; Fig. $\left.6 \mathrm{~B}\right)$ or social novelty preference $\left(t_{(12)}=0.10, p=0.92\right.$; unpaired Student's $t$-test; Fig. $\left.6 \mathrm{C}\right)$ in hM4D(Gi)-mCherryexpressed mice. However, a statistically significant difference was observed between vehicle- $(n=7)$ and CNO-treated hM4D(Gi)-mCherry-expressed mice $(n=7)$ in discrimination index of 7-day long-term SRM $\left(t_{(12)}=3.66, p=0.003\right.$; unpaired Student's $t$-test; Fig. 6D).

To establish whether dCA2 promotes long-term SRM through its outputs to dLS, we bilaterally injected $\mathrm{AAV}_{5}$-hSyn-DIO-hM4D(Gi)-mCherry into dCA2 of Amigo2-Cre mice. After allowing two weeks for viral expression, mice were implanted with cannula targeting dLS. One week later, mice underwent threechamber paradigm test. Twenty minutes before sociability test, mice were injected with vehicle or CNO via cannula targeting dLS. Post hoc histological examination of brain sections revealed robust coexpression of mCherry with STEP in dCA2 (Fig. 7A, bottom left panel). We also observed dense projection fibers from dCA2 to dLS (Fig. 7A, bottom right panel). We found that CNO-treated hM4D(Gi)-mCherryexpressed mice $(n=7)$ performed similarly to vehicle-treated hM4D(Gi)-mCherry-expressed mice $(n=7)$ in discrimination index of sociability $\left(t_{(12)}=1.15, p=0.27\right.$; unpaired Student's $t$-test; Fig. 7B), social novelty preference $\left(t_{(12)}=1.54, p=0.15\right.$; unpaired Student's $t$-test; Fig. $\left.7 \mathrm{C}\right)$ and 7-day long-term SRM $\left(t_{(12)}=1.27, p\right.$ $=0.23$; unpaired Student's $t$-test; Fig. 7D).

\section{Activation of dCA2-to-vCA1 projections ameliorates long-term SRM deficit in $0 \mathrm{xtr}^{-/-}$mice}

Activation of OXTRs in the CA2 is known to increase neuronal excitability [32]. To investigate whether specific aspect of long-term SRM deficit in Oxtr ${ }^{-/-}$mice is indeed due to deficits in CA2 function within the dCA2-to-vCA1 circuit, we examined if the selective chemogenetic activation of the dCA2-to-vCA1 circuit could rescue long-term SRM deficit. To do this, we performed bilateral injections of control $\mathrm{AAV}_{5^{-}}$ hSyn-DIO-mCherry or AAV $5_{5}$-hSyn-DIO-hM3D(Gq)-mCherry into dCA2 of $\mathrm{Oxtr}^{-1-}$ mice. After allowing two weeks for viral expression, mice were implanted with cannula targeting vCA1. One week later, mice underwent three-chamber paradigm test. Twenty minutes before sociability test, mice were injected with CNO via cannula targeting vCA1 (Fig. 8A). Post hoc histological examination of brain sections revealed robust co-expression of mCherry with STEP in dCA2 (Fig. 8A, bottom left panel). We also observed dense projection fibers from dCA2 to vCA1 (Fig. 8A, bottom right panel). The hM3D(Gq)-mCherry-expressed 
$\mathrm{Oxtr}^{-1-}$ mice $(n=7)$ performed similarly to mCherry-expressed $\mathrm{Oxtr}^{-1-}$ mice $(n=7)$ in discrimination index of sociability $\left(t_{(12)}=1.89, p=0.08\right.$; unpaired Student's $t$-test; Fig. $\left.8 \mathrm{~B}\right)$ and social novelty preference $\left(t_{(12)}=\right.$ $0.45, p=0.66$; unpaired Student's $t$-test; Fig. $8 C$ ). In 7-day long-term SRM test, a statistically significant discrimination index was observed between mCherry-expressed and hM3D(Gq)-mCherry-expressed Oxtr ${ }^{-1}$ - mice in 7-day long-term SRM $\left(t_{(12)}=2.37, p=0.03\right.$; unpaired Student's $t$-test; Fig. 8D). Together, these findings suggest that dCA2 promotes the persistence of long-term SRM, at least in part, through its projections to vCA1. Remarkably, activation of the dCA2-to-vCA1 circuit can effectively ameliorate longterm SRM deficit in $\mathrm{Oxtr}^{-/-}$mice.

\section{Discussion}

SRM is considered a hippocampus-dependent memory and can persist over long periods of time following successful encoding [7, 33]. In this study, using three-chamber paradigm test to measure longterm SRM in Amigo2-Cre mediated Oxtr ${ }^{-1-}$ mice, we show that endogenous OXTR signaling in dCA2 plays an essential role in regulating the persistence of long-term SRM. Through chemogenetic terminal-specific manipulations, we identify the contribution of dCA2 and its projections to vCA1 in the formation of longterm SRM. More importantly, targeted activation of dCA2-to-vCA1 projections effectively ameliorates longterm SRM deficit observed in $\mathrm{Oxtr}^{-1-}$ mice. Collectively, these findings expand on previous studies linking dCA2 function to SRM $[9,13-15]$ and support the notion that CA2 OXTR signaling is crucial for the longterm persistence of SRM [12]. To the best of our knowledge, this is the first study aiming to uncover the functional role of the dCA2-to-vCA1 circuit in mediating endogenous OXTergic modulation of long-term SRM formation.

Rodents can retain SRM for at least one week by group housing $[3,34,35]$. In contrast, SRM persists no more than a few hours in socially isolated animals $[15,36]$. These findings strongly indicate that there exist group housing-related factors prolonging an hour-long labile SRM to days. The potential contributors are neuromodulators, which are employed in SRM formation [33, 37]. The hippocampal CA2 region has been proven to be a crucial hub in SRM processing $[9,13]$. A host of neuromodulatory inputs have been suggested to converge on the CA2 subfield [38, 39], among which OXT and its closely related neuropeptide arginine vasopressin have been received much more attention. We have previously reported that conditional deletion of CA2/CA3a OXTR impairs the persistence of long-term SRM [12], but no consensus has thus far emerged. However, Chiang and coworkers [22] have shown that silencing dCA3 had no significant effect on SRM. Because the CA2 heavily innervates the CA3a [28], this anatomical connectivity might bias interpretation of the role of CA3a OXTRs in governing long-term SRM. Unfortunately, the Cre/loxP conditional knockout strategy used in our previous study did not reach selective deletion of Oxtrfrom CA2 or CA3a neurons. This shortcoming can be overcome with the aid of Amigo2-Cre mice, in which Cre expression is largely restricted to CA2 pyramidal neurons [9]. Although one previous study showed expression of Amigo 2 mRNA not only in the CA2 but also in some CA3a cells in mouse brain [40], we found this rare. In the present study, by taking advantage of this transgenic mouse line that enables precise deletion of CA2 Oxtr, we focused our research on the regulatory role of 
endogenous OXTR signaling in dCA2 and its outputs in controlling long-term SRM persistence. In support, we clearly demonstrate that mice lacking CA2 OXTRs have a deficit in the persistence of long-term SRM and, in particular, chemogenetic activation of OXT neurons in the PVN enhances long-term SRM, an effect that was blocked by local application of an OXTR antagonist L-368,899 in dCA2. In addition, we find no changes in sociability and social novelty preference in $\mathrm{Oxtr}^{-1-}$ mice, suggesting a specific role for dCA2 OXTR signaling in long-term SRM. Consistent with our previous work [12], we show that memory retention was unaffected at 1 day after encoding but significantly impaired at 7 days after the initial interaction in mice lacking CA2 Oxtr, supporting that OXTR signaling in the CA2 is specifically critical for the persistence of long-term SRM. We, therefore, speculate that OXTR signaling in ICA2 during SRM encoding may strengthen the salience of social information and enable this information to be consolidated into a long-term form, dissociating decay of memory traces with the passage of time. It is known that long-term SRM is dependent on protein synthesis and CAMP responsive element binding protein activity [7]; thus, it seems likely that OXTR signaling might exert effects via similar cellular mechanisms to promote the persistence of long-term SRM, which needs to be investigated in the future.

Our findings reinforce the importance of dCA2 neuronal activity in long-term SRM formation. Indeed, we demonstrate that chemogenetic silencing of dCA2 pyramidal neurons impairs long-term SRM. This finding aligns with a previous study demonstrating that excitotoxic $N$-methyl-D-aspartate lesions of the CA2 impair SRM in mice [14]. Similarly, Hitti and Siegelbaum [9] showed that chronic genetically engineered shutdown of CA2 pyramidal cell synaptic transmission with tetanus neurotoxin also results in an impairment of SRM without affecting sociability. Furthermore, acute chemogenetic or optogenetic silencing dCA2 impaired SRM processing in Amigo2-Cre mice infected in dCA2 with AAV ${ }_{2}$-hSyn-DIOhM4D(Gi)-mCitrine or AAV 2/5-EF1a-DIO-eArch3.0-eYFP, respectively [13]. A pressing question that follows these observations is how dCA2 contributes to SRM formation. In rodents, SRM is mediated mainly by olfactory cues perceived via the olfactory system [41]. Considering that the CA2 receives non-spatial information from the entorhinal cortex $[28,42,43]$ and the entorhinal cortex receives extensive inputs from the olfactory bulb and piriform cortex, both areas that devote to processing of olfactory information [44], it is therefore possible that the CA2 may contribute to SRM by incorporating these social and olfactory inputs to create a memory trace necessary for the recognition process [37].

Our characterization of an excitatory pathway from dCA2 to vCA1 may account for endogenous OXTergic modulation of long-term SRM formation. The dynamic activity of this projection has been implicated in processing of SRM [13]. While dCA2 also sends anatomical projections to dLS, which was recently shown to participate in promoting social aggression [29], we did not observe any effect of terminal-specific silencing of dCA2-to-dLS projections on long-term SRM. More importantly, we provide evidence that acute activation of the dCA2-to-vCA1 circuit can effectively improve long-term SRM deficit observed in $\mathrm{Oxtr}^{-/-}$ mice. Thus, interplay within OXTR signaling-modulated dCA2-to-vCA1 circuit likely plays a specific role in long-term SRM formation. Notably, a previous study has shown that vCA1 and its projections to the nucleus accumbens (NAc) shell play a necessary and sufficient role in storing SRM [45], raising an 
intriguing possibility that dCA2 targets vCA1 pyramidal neurons that project to the NAc shell to subserve long-term SRM storage. Additional studies are needed to clarify this issue.

In conclusion, our results integrate CA2 OXTR signaling into the dCA2-to-vCA1 circuit to promote the persistence of long-term SRM. Our findings contribute to a better understanding of neuromodulatory and neural circuit mechanisms contributing to long-term SRM formation. Relevant information may provide a novel therapeutic avenue to the treatment of social cognitive disabilities seen in autism spectrum disorders and other psychiatric disorders [46-48].

\section{Declarations}

\section{Acknowledgements}

The authors thank Dr. Katsuhiko Nishimori for kindly providing Oxtr $\mathrm{Venus-Neo/+}^{\mathrm{mice}}$. The authors also thank the technical services provided by the Bio-image Core Facility of the National Core Facility Program for Biotechnology, Ministry of Science and Technology, Taiwan.

\section{Funding}

This work was supported by research grants from the National Health Research Institute (NHRI-EX11010912NI and NHRI-EX111-10912NI), the Ministry of Science and Technology (107-2320-B-006-037-MY3, 109-2320-B-006-039-MY3 and 110-2331-B-006-036-MY3), Taiwan.

\section{Conflict of interest}

The authors declare no conflict of interest.

\section{Author contributions}

T.C.T., Y.S.F., Y.C.H., L.C.H. and K.S.H. designed experiments; T.C.T., Y.S.F. and Y.C.H. performed experiments and analyzed data; T.C.T., Y.S.F., L.C.H. and K.S.H. wrote the paper.

\section{Data availability}

The authors confirm that all data generated and analyzed during this study are either included in this published article or available from the corresponding authors upon reasonable request.

\section{Ethics approval}

All experimental procedures were approved by the Institutional Animal Care and Use Committee (IACUC) at the National Cheng Kung University (authorization \#107023).

\section{Consent to participate}

Not applicable. 


\section{Consent to publish}

Not applicable.

\section{References}

1. Markham JA, Juraska JM (2007) Social recognition memory: influence of age, sex, and ovarian hormonal status. Physiol Behav 92:881-888. https://doi.org/10.1016/j.physbeh.2007.06.020.

2. McGraw LA, Young LJ (2010) The prairie vole: an emerging model organism for understanding the social brain. Trends Neurosci 33:103-109. https://doi.org/ 10.1016/j.tins.2009.11.006.

3. Okuyama T, Yokoi S, Abe H et al (2014) A neural mechanism underlying mating preferences for familiar individuals in medaka fish. Science 343:91-94. https://doi.org/10.1126/science.1244724.

4. Ferguson JN, Young LJ, Insel TR (2002) The neuroendocrine basis of social recognition. Front Neuroendocrinol 23:200-224. https://doi.org/ 10.1006/frne.2002.0229.

5. Jacobs SA, Huang F, Tsien JZ, Wei W (2016) Social recognition memory test in rodents. Bio Protoc 6 : e1804. https://doi.org/10.21769/BioProtoc.3120.

6. de la Zerda SH, Netser S, Magalnik H et al (2020) Social recognition in rats and mice requires integration of olfactory, somatosensory and auditory cues. Preprint at bioRxiv. https://doi.org/10.1101/2020.05.05.078139.

7. Kogan JH, Frankland PW, Silva AJ (2000) Long-term memory underlying hippocampus-dependent social recognition in mice. Hippocampus 10:47-56. https://doi.org/10.1002/(SICI)10981063(2000)10:1<47::AID-HIP05>3.0.C0;2-6.

8. Suzuki A, Fukushima H, Mukawa T et al (2011) Upregulation of CREB-mediated transcription enhances both short- and long-term memory. J Neurosci 31:8786-8802. https://doi.org/ 10.1523/JNEUROSCI.3257-10.2011.

9. Hitti FL, Siegelbaum SA (2014) The hippocampal CA2 region is essential for social memory. Nature 508:88-92. https://doi.org/10.1038/nature13028.

10. Garrido Zinn C, Clairis N et al (2016) Major neurotransmitter systems in dorsal hippocampus and basolateral amygdala control social recognition memory. Proc Natl Acad Sci USA 113:E4914-E4919. https://doi.org/ 10.1073/pnas.1609883113.

11. Tanimizu T, Kenney JW, Okano E et al (2017) Functional connectivity of multiple brain regions required for the consolidation of social recognition memory. J Neurosci 37:4103-4116. https://doi.org/ 10.1523/JNEUROSCI.3451-16.2017.

12. Lin YT, Hsieh TY, Tsai TC et al (2018) Conditional deletion of hippocampal CA2/CA3a oxytocin receptors impairs the persistence of long-term social recognition memory in mice. J Neurosci 38:1218-1231. https://doi.org/ 10.1523/JNEUROSCI.1896-17.2017. 
13. Meira T, Leroy F, Buss EW et al (2018) A hippocampal circuit linking dorsal CA2 to ventral CA1 critical for social memory dynamics. Nat Commun 9:4163. https://doi.org/ 10.1038/s41467-018-06501-w.

14. Stevenson EL, Caldwell HK (2014) Lesions to the CA2 region of the hippocampus impair social memory in mice. Eur J Neurosci 40:3294-3301. https://doi.org/ 10.1111/ejn.12689.

15. Okuyama T, Kitamura T, Roy DS et al (2016) Ventral CA1 neurons store social memory. Science 353:1536-1541. https://doi.org/ 10.1126/science.aaf7003.

16. Jurek B, Neumann ID (2018) The oxytocin receptor: from intracellular signaling to behavior. Physiol Rev 98:1805-1908. https://doi.org/ 10.1152/physrev.00031.2017.

17. Froemke RC, Young LJ (2021) Oxytocin, neural plasticity, and social behavior. Annu Rev Neurosci 44:359-381. https://doi.org/ 10.1146/annurev-neuro-102320-102847.

18. Mitre M, Kranz TM, Marlin BJ et al (2017) Sex-specific differences in oxytocin receptor expression and function for parental behavior. Gend Genome 1:142-166. https://doi.org/ 10.1089/gg.2017.0017.

19. Grinevich V, Stoop R (2018) Interplay between oxytocin and sensory systems in the orchestration of socio-emotional behaviors. Neuron 99:887-904. https://doi.org/ 10.1016/j.neuron.2018.07.016.

20. Zhang J, Li SJ, Miao W et al (2021) Oxytocin regulates synaptic transmission in the sensory cortices in a developmentally dynamic manner. Front Cell Neurosci 15:673439. https://doi.org/10.3389/fncel.2021.673439.

21. Raam T, McAvoy KM, Besnard A et al (2017) Hippocampal oxytocin receptors are necessary for discrimination of social stimuli. Nat Commun 8:2001. https://doi.org/ 10.1038/s41467-017-02173-0.

22. Chiang MC, Huang AJY, Wintzer ME et al (2018) A role for CA3 in social recognition memory. Behav Brain Res 354:22-30. https://doi.org/ 10.1016/j.bbr.2018.01.019.

23. Yoshida $M$, Takayanagi $Y$, Inoue $K$ et al (2009) Evidence that oxytocin exerts anxiolytic effects via oxytocin receptor expressed in serotonergic neurons in mice. J Neurosci 29:2259-2271. https://doi.org/ 10.1523/JNEUROSCI.5593-08.2009.

24. Lin YT, Chen CC, Huang CC et al (2017) Oxytocin stimulates hippocampal neurogenesis via oxytocin receptor expressed in CA3 pyramidal neurons. Nat Commun 8:537. https://doi.org/ 10.1038/s41467017-00675-5.

25. Stachniak TJ, Ghosh A, Sternson SM (2014) Chemogenetic synaptic silencing of neural circuits localizes a hypothalamus $\rightarrow$ midbrain pathway for feeding behavior. Neuron 82:797-808. https://doi.org/ 10.1016/j.neuron.2014.04.008.

26. Chiou CS, Chen CC, Tsai TC et al (2016) Alleviating bone cancer-induced mechanical hypersensitivity by inhibiting neuronal activity in the anterior cingulate cortex. Anesthesiology 125:779-792. https://doi.org/ 10.1097/ALN.0000000000001237.

27. Shinohara Y, Hosoya A, Yahagi K et al (2012) Hippocampal CA3 and CA2 have distinct bilateral innervation patterns to CA1 in rodents. Eur J Neurosci 35:702-710. https://doi.org/ 10.1111/j.14609568.2012.07993.x. 
28. Kohara K, Pignatelli M, Rivest AJ et al (2014) Cell type-specific genetic and optogenetic tools reveal hippocampal CA2 circuits. Nat Neurosci 17:269-279. https://doi.org/ 10.1038/nn.3614.

29. Leroy F, Park J, Asok A et al (2018) A circuit from hippocampal CA2 to lateral septum disinhibits social aggression. Nature 564:213-218. https://doi.org/ 10.1038/s41586-018-0772-0.

30. Moy SS, Nadler JJ, Perez A et al (2004) Sociability and preference for social novelty in five inbred strains: an approach to assess autistic-like behavior in mice. Genes Brain Behav 3:287-302. https://doi.org/ 10.1111/j.1601-1848.2004.00076.x.

31. Ferretti V, Maltese F, Contarini G et al (2019) Oxytocin signaling in the central amygdala modulates emotion discrimination in mice. Curr Biol 29:1938-1953. https://doi.org/ 10.1016/j.cub.2019.04.070.

32. Tirko NN, Eyring KW, Carcea I et al (2018) Oxytocin transforms firing mode of CA2 hippocampal neurons. Neuron 100:593-608. https://doi.org/ 10.1016/j.neuron.2018.09.008.

33. Gur R, Tendler A, Wagner S (2014) Long-term social recognition memory is mediated by oxytocindependent synaptic plasticity in the medial amygdala. Biol Psychiatry 76:377-386. https://doi.org/ 10.1016/j.biopsych.2014.03.022.

34. Bluthé RM, Gheusi G, Dantzer R (1993) Gonadal steroids influence the involvement of arginine vasopressin in social recognition in mice. Psychoneuroendocrinology 18:323-335. https://doi.org/ 10.1016/0306-4530(93)90028-j.

35. Smith AS, Williams Avram SK et al (2016) Targeted activation of the hippocampal CA2 area strongly enhances social memory. Mol Psychiatry 21:1137-1144. https://doi.org/ 10.1038/mp.2015.189.

36. Shahar-Gold H, Gur R, Wagner S (2013) Rapid and reversible impairments of short- and long-term social recognition memory are caused by acute isolation of adult rats via distinct mechanisms. PLoS One 8:e65085. https://doi.org/ 10.1371/journal.pone.0065085.

37. Tzakis N, Holahan MR (2019) Social memory and the role of the hippocampal CA2 region. Front Behav Neurosci 13:233. https://doi.org/ 10.3389/fnagi.2019.00253.

38. Benoy A, Dasgupta A, Sajikumar S (2018) Hippocampal area CA2: an emerging modulatory gateway in the hippocampal circuit. Exp Brain Res 236:919-931. https://doi.org/ 10.1007/s00221-018-5187-5.

39. Lehr AB, Kumar A, Tetzlaff $C$ et al (2021) CA2 beyond social memory: evidence for a fundamental role in hippocampal information processing. Neurosci Biobehav Rev 126:398-412. https://doi.org/ 10.1016/j.neubiorev.2021.03.020.

40. Laeremans A, Nys J, Luyten W et al (2013) AMIGO2 mRNA expression in hippocampal CA2 and CA3a. Brain Struct Funct 218:123-130. https://doi.org/ 10.1007/s00429-012-0387-4.

41. Insel TR, Fernald RD (2004) How the brain processes social information: searching for the social brain. Annu Rev Neurosci 27:697-722. https://doi.org/ 10.1146/annurev.neuro.27.070203.144148.

42. Chevaleyre V, Siegelbaum SA (2010) Strong CA2 pyramidal neuron synapses define a powerful disynaptic cortico-hippocampal loop. Neuron 66:560-572. https://doi.org/ 10.1016/j.neuron.2010.04.013. 
43. Dudek SM, Alexander GM, Farris S (2016) Rediscovering area CA2: unique properties and functions. Nat Rev Neurosci 17:89-102. https://doi.org/ 10.1038/nrn.2015.22.

44. Boeijinga PH, Van Groen T (1984) Inputs from the olfactory bulb and olfactory cortex to the entorhinal cortex in the cat. II. Physiological studies. Exp Brain Res 57:40-48. https://doi.org/ 10.1007/BF00231130.

45. Okuyama T, Kitamura T, Roy DS et al (2016) Ventral CA1 neurons store social memory. Science 353:1536-1541. https://doi.org/ 10.1126/science.aaf7003.

46. Kennedy DP, Adolphs R (2012) The social brain in psychiatric and neurological disorders. Trends Cogn Sci 16:559-572. https://doi.org/ 10.1016/j.tics.2012.09.006.

47. Allsop SA, Vander Weele CM, Wichmann R, Tye KM (2014) Optogenetic insights on the relationship between anxiety-related behaviors and social deficits. Front Behav Neurosci 8:241. https://doi.org/ 10.3389/fnbeh.2014.00241.

48. Pasciuto E, Borrie SC, Kanellopoulos AK et al (2015) Autism spectrum disorders: translating human deficits into mouse behavior. Neurobiol Learn Mem 124:71-87. https://doi.org/ 10.1016/j.nlm.2015.07.013.

\section{Figures}

\section{Figure 1}

Deletion of Oxtr in CA2 excitatory neurons of the mouse hippocampus. A Doubled-labeled confocal immunofluorescent images showing the colocalization of OXTRs (green) and STEP (red) in dCA2 of the mouse hippocampus. The inserts represent high magnification of the boxed area. Scale bar: $50 \mathrm{~mm}$. SO, stratum oriens; SP, stratum pyramidale; SR, stratum radiatum. B PCR screening of tail-derived genomic DNA for selection of Oxtr/- mice. C Dual-probe FISH images showing the expression of OxtrmRNA and Amigo 2 mRNA in dCA2 of WT and Oxtr ${ }^{-1}$ mice (counterstained with DAPI, blue). Scale bar, $50 \mathrm{~mm}$. Data was replicated in 4 mice. D Representative images with cresyl violet staining of dCA2 showing that the number of pyramidal neurons was not affected by targeted deletion of Oxtr compared with age-matched WT mice. Group data showing the summary results from 3-4 mice of each group at 12 weeks old. Scale bars: left, $500 \mathrm{~mm}$; right, $50 \mathrm{~mm}$. The total number of animal examined is indicated by $n$ in parenthesis. Error bars represent the SEM. 
A
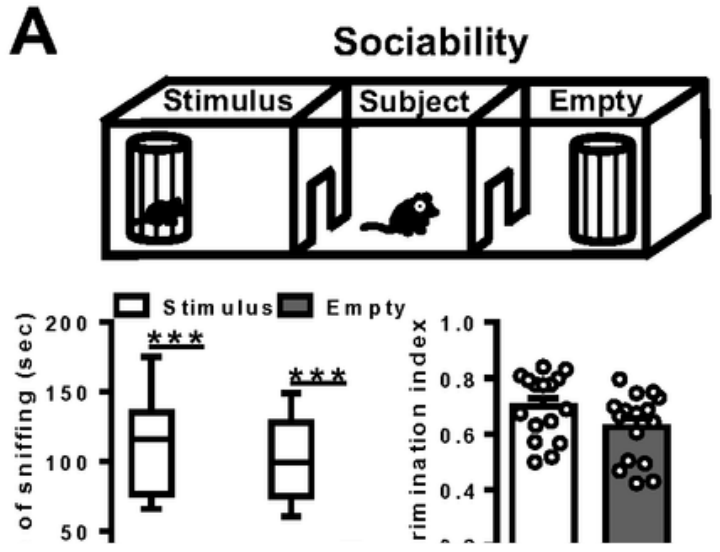

B
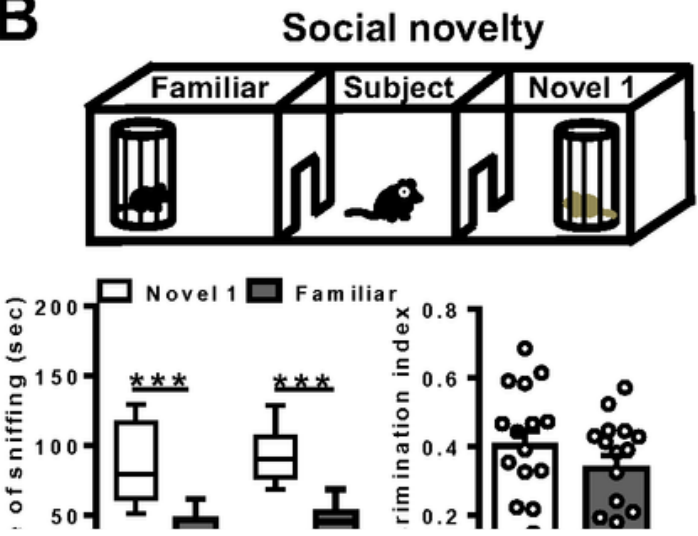

Figure 2

Deletion of Oxtr in CA2 excitatory neurons leads to impaired persistence long-term SRM. A Top, schematic representation of the three-chamber sociability test. Bottom left, time spent by the subject mouse in sniffing directed at a wire cage containing the juvenile stimulus mouse or an empty wire cage. Both WT and $\mathrm{Oxtr}^{\mathrm{r}}$ - subject mice spent significantly more time interacting with the wire cage containing the juvenile stimulus mouse than the empty wire cage. Bottom right, discrimination index (stimulus minus 
empty) was similar between WT and Oxtr ${ }^{-/}$subject mice in the sociability test. B Top, schematic representation of the three-chamber social novelty preference test. Bottom left, time spent by the subject mouse in sniffing directed at the wire cage containing a familiar mouse or a novel 1 mouse, 10 min after the sociability test. Both WT and $O x t r^{-/}$subject mice spent significantly more time sniffing the cage containing the novel mouse than the familiar mouse. Bottom right, discrimination index (novel 1 minus familiar) was comparable between WT and $O x t r^{2 /-}$ subject mice in the social novelty preference test. C Top, schematic representation of the three-chamber long-term SRM test. Bottom left, time spent by the subject mouse in sniffing directed at the wire cage containing a familiar mouse or a novel 2 mouse, 1 day after the initial interaction. Both WT and $O x t r^{-/}$subject mice spent significantly more time sniffing the cage containing the novel mouse than the familiar mouse. Bottom right, discrimination index (novel 2 minus familiar) was similar between WT and $O x t r^{-/}$subject mice in 1-day long-term SRM test. D Top, schematic representation of the three-chamber long-term SRM test. Bottom, time spent by the subject mouse in sniffing directed at the wire cage containing a familiar mouse or a novel 2 mouse, 7 days after the initial interaction. WT, but not $O x t r r^{-}$, subject mice spent significantly more time sniffing the cage containing the novel mouse than the familiar mouse. Bottom right, discrimination index (novel 2 minus familiar) of $\mathrm{Oxtr}^{-/}$subject mice was significantly less than WT subject mice in 7-day long-term SRM test. The total number of animal examined is indicated by $n$ in parenthesis. Error bars represent the SEM; ${ }^{\star} p<0.05,{ }^{\star \star} p<0.01,{ }^{* \star *} p<0.001$.

\section{Figure 3}

Activation of PVN OXT neurons enhance long-term SRM. A Schematic representation of viral injection and $\mathrm{CNO}$ administration. Three weeks after stereotaxic injection of $\mathrm{AAV}_{5}-\mathrm{mOXT}$-hM3D(Gq)-mCherry into the PVN, mice were subjected to three-chamber paradigm test and long-term SRM retention was tested 7 days after the initial interaction. Mice were bilaterally injected with either vehicle (Veh) or CNO into dCA2 20 min before the initial interaction. Representative images showing the co-expression of hM3D(Gq)mCherry and OXT immunoreactivity in the PVN. Scale bar represents $50 \mathrm{~mm}$. B Top, schematic representation of the three-chamber sociability test. Bottom left, time spent by the subject mouse in sniffing directed at the wire cage containing the juvenile stimulus mouse or the empty wire cage. $\mathrm{hM} 3 \mathrm{D}(\mathrm{Gq}) /$ Veh and $\mathrm{hM} 3 \mathrm{D}(\mathrm{Gq}) / \mathrm{CNO}$ subject mice spent significantly more time interacting with the wire cage containing the juvenile stimulus mouse than the empty wire cage. Bottom right, discrimination index (stimulus minus empty) was similar among groups in the sociability test. C Top, schematic representation of the three-chamber social novelty preference test. Bottom left, time spent by the subject mouse in sniffing directed at the wire cage containing a familiar mouse or a novel 1 mouse, $10 \mathrm{~min}$ after the sociability test. $\mathrm{hM} 3 \mathrm{D}(\mathrm{Gq}) / \mathrm{Veh}$ and $\mathrm{hM} 3 \mathrm{D}(\mathrm{Gq}) / \mathrm{CNO}$ subject mice spent significantly more time sniffing the cage containing the novel mouse than the familiar mouse. Bottom right, discrimination index (novel 1 minus familiar) was comparable among groups in the social novelty preference test. D Top, schematic representation of the three-chamber long-term SRM test. Bottom, time spent by the subject 
mouse in sniffing directed at the wire cage containing a familiar mouse or a novel 2 mouse, 7 days after the initial interaction. $\mathrm{hM} 3 \mathrm{D}(\mathrm{Gq}) / \mathrm{Veh}$ and $\mathrm{hM} 3 \mathrm{D}(\mathrm{Gq}) / \mathrm{CNO}$ subject mice spent significantly more time sniffing the cage containing the novel mouse than the familiar mouse. Bottom right, discrimination index (novel 2 minus familiar) of hM3D(Gq)/CNO subject mice was significantly higher than those of $\mathrm{hM} 3 \mathrm{D}(\mathrm{Gq}) /$ Veh subject mice in 7-day long-term SRM test. The total number of animal examined is indicated by $n$ in parenthesis. Error bars represent the SEM; ${ }^{\star \star} p<0.01, * \star \star x<0.001$.

\section{Figure 4}

Pharmacological antagonism of OXTR in dCA2 blocks chemogenetic enhancement of long-term SRM. A Schematic representation of viral injection and CNO administration. Three weeks after stereotaxic injection of $\mathrm{AAV}_{5}$-mOXT-hM3D(Gq)-mCherry into the PVN, mice were subjected to three-chamber paradigm test and long-term SRM retention was tested 7 days after the initial interaction. Mice were bilaterally administered of vehicle (Veh) or an OXTR antagonist L-368,899 into dCA2 10 min before CNO injection, followed by three-chamber paradigm test $20 \mathrm{~min}$ after $\mathrm{CNO}$ injection. Representative images showing the co-expression of hM3D(Gq)-mCherry and OXT immunoreactivity in the PVN. mCherry signals of axonal projections were observed in dCA2. Scale bar represents $50 \mathrm{~mm}$. B Top, schematic representation of the three-chamber sociability test. Bottom left, time spent by the subject mouse in sniffing directed at the wire cage containing the juvenile stimulus mouse or the empty wire cage. $\mathrm{hM} 3 \mathrm{D}(\mathrm{Gq}) / \mathrm{CNO} / \mathrm{Veh}$ and $\mathrm{hM} 3 \mathrm{D}(\mathrm{Gq}) / \mathrm{CNO} / \mathrm{L}-368,899$ subject mice spent significantly more time interacting with the wire cage containing the juvenile stimulus mouse than the empty wire cage. Bottom right, discrimination index (stimulus minus empty) was similar between groups in the sociability test. C Top, schematic representation of the three-chamber social novelty preference test. Bottom left, time spent by the subject mouse in sniffing directed at the wire cage containing a familiar mouse or a novel 1 mouse, 10 min after the sociability test. hM3D(Gq)/CNO/Veh and hM3D(Gq)/CNO/L-368,899 subject mice spent significantly more time sniffing the cage containing the novel mouse than the familiar mouse. Bottom right, discrimination index (novel 1 minus familiar) was comparable between groups in the social novelty preference test. D Top, schematic representation of the three-chamber long-term SRM test. Bottom, time spent by the subject mouse in sniffing directed at the wire cage containing a familiar mouse or a novel 2 mouse, 7 days after the initial interaction. hM3D(Gq)/CNO/Veh, but not $\mathrm{hM} 3 \mathrm{D}(\mathrm{Gq}) / \mathrm{CNO} / \mathrm{L}-368,899$, subject mice spent significantly more time sniffing the cage containing the novel mouse than the familiar mouse. Bottom right, discrimination index (novel 2 minus familiar) of $\mathrm{hM} 3 \mathrm{D}(\mathrm{Gq}) / \mathrm{CNO} /$ Veh subject mice was significantly higher than that of $\mathrm{hM} 3 \mathrm{D}(\mathrm{Gq}) / \mathrm{CNO} / \mathrm{L}-368,899$ subject mice in 7-day long-term SRM test. The total number of animal examined is indicated by $n$ in parenthesis. Error bars represent the SEM; ${ }^{\star} p<0.05,{ }^{* \star} p<0.01,{ }^{\star \star \star} p<0.001$.

\section{Figure 5}


dCA2 neuronal activity is necessary for the persistence of long-term SRM. $\boldsymbol{A}$, Schematic representation of

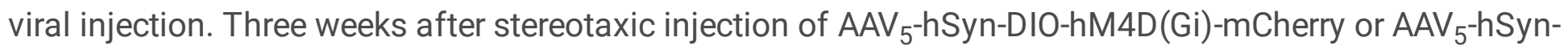
DIO-mCherry into the dCA2 of Amigo2-Cre mice, mice were subjected to three-chamber paradigm test and long-term SRM retention was tested 7 days after the initial interaction. Mice were intraperitoneally injected with CNO 30 min before the initial interaction. Representative images showing the co-expression of hM4D(Gi)-mCherry and STEP immunoreactivity in dCA2 pyramidal neurons. Scale bar represents 50 $\mathrm{mm}$. B Top, schematic representation of the three-chamber sociability test. Bottom left, time spent by the subject mouse in sniffing directed at the wire cage containing the juvenile stimulus mouse or the empty wire cage. Both $\mathrm{hM} 4 \mathrm{D}(\mathrm{Gi}) / \mathrm{CNO}$ and mCherry/CNO subject mice spent significantly more time interacting with the wire cage containing the juvenile stimulus mouse than the empty wire cage. Bottom right, discrimination index (stimulus minus empty) was similar between groups in the sociability test. C Top, schematic representation of the three-chamber social novelty preference test. Bottom left, time spent by the subject mouse in sniffing directed at the wire cage containing a familiar mouse or a novel 1 mouse, 10 min after the sociability test. Both hM4D(Gi)/CNO and mCherry/CNO subject mice spent significantly more time sniffing the cage containing the novel mouse than the familiar mouse. Bottom right, discrimination index (novel 1 minus familiar) was comparable between groups in the social novelty preference test. D Top, schematic representation of the three-chamber long-term SRM test. Bottom, time spent by the subject mouse in sniffing directed at the wire cage containing a familiar mouse or a novel 2 mouse, 7 days after the initial interaction. mCherry/CNO, but not hM4D(Gi)/CNO, subject mice spent significantly more time sniffing the cage containing the novel mouse than the familiar mouse. Bottom right, discrimination index (novel 2 minus familiar) of hM4D(Gi)/CNO subject mice was significantly less than $\mathrm{mCherry/CNO}$ subject mice in 7-day long-term SRM test. The total number of animal examined is indicated by $n$ in parenthesis. Error bars represent the SEM; ${ }^{* \star} p<0.01$, ${ }^{* \star *} p<0.001$.

\section{Figure 6}

dCA2 projections to vCA1 is necessary for long-term SRM. A Schematic representation of viral injection and $\mathrm{CNO}$ administration. Three weeks after stereotaxic injection of $\mathrm{AAV}_{5}$-hSyn-DIO-hM4D(Gi)-mCherry into the dCA2 of Amigo2-Cre mice, mice were subjected to three-chamber paradigm test and long-term SRM retention was tested 7 days after the initial interaction. Mice were bilaterally administered of vehicle (Veh) or CNO into vCA1 20 min before the initial interaction. Representative images showing the coexpression of hM4D(Gi)-mCherry and STEP immunoreactivity in dCA2 pyramidal neurons. Scale bars represent $50 \mathrm{~mm}$. mCherry signals of axonal projections were observed in vCA1. Scale bar represents $100 \mathrm{~mm}$. NeuN, neuronal nuclear protein; SO, stratum oriens; SP, stratum pyramidale; SR, stratum radiatum. B Top, schematic representation of the three-chamber sociability test. Bottom left, time spent by the subject mouse in sniffing directed at the wire cage containing the juvenile stimulus mouse or the empty wire cage. Both $\mathrm{hM} 4 \mathrm{D}(\mathrm{Gi}) / \mathrm{Veh}$ and $\mathrm{hM} 4 \mathrm{D}(\mathrm{Gi}) / \mathrm{CNO}$ subject mice spent significantly more time interacting with the wire cage containing the juvenile stimulus mouse than the empty wire cage. Bottom right, discrimination index (stimulus minus empty) was similar between hM4D(Gi)/Veh and 
$\mathrm{hM} 4 \mathrm{D}(\mathrm{Gi}) / \mathrm{CNO}$ subject mice in the sociability test. C Top, schematic representation of the three-chamber social novelty preference test. Bottom left, time spent by the subject mouse in sniffing directed at the wire cage containing a familiar mouse or a novel 1 mouse, 10 min after the sociability test. Both $\mathrm{hM} 4 \mathrm{D}(\mathrm{Gi}) / \mathrm{Veh}$ and hM4D(Gi)/CNO subject mice spent significantly more time sniffing the cage containing the novel mouse than the familiar mouse. Bottom right, discrimination index (novel 1 minus familiar) was comparable between hM4D(Gi)/Veh and hM4D(Gi)/CNO subject mice in the social novelty preference test. D Top, schematic representation of the three-chamber long-term SRM test. Bottom, time spent by the subject mouse in sniffing directed at the wire cage containing a familiar mouse or a novel 2 mouse, 7 days after the initial interaction. Both $\mathrm{hM} 4 \mathrm{D}(\mathrm{Gi}) /$ Veh and $\mathrm{hM} 4 \mathrm{D}(\mathrm{Gi}) / \mathrm{CNO}$ subject mice spent significantly more time sniffing the cage containing the novel mouse than the familiar mouse. Bottom right, discrimination index (novel 2 minus familiar) of $\mathrm{hM} 4 \mathrm{D}(\mathrm{Gi}) / \mathrm{CNO}$ subject mice was significantly less than $\mathrm{hM} 4 \mathrm{D}(\mathrm{Gi}) /$ Veh subject mice in 7-day long-term SRM test. The total number of animal examined is indicated by $n$ in parenthesis. Error bars represent the SEM; ${ }^{\star \star} p<0.01$, ${ }^{\star \star \star} p<0.001$.

\section{Figure 7}

dCA2 projections to dLS is not involved in long-term SRM. A Schematic representation of viral injection. Three weeks after stereotaxic injection of $\mathrm{AAV}_{5}$-hSyn-DIO-hM4D(Gi)-mCherry into the dCA2 of Amigo2Cre mice, mice were subjected to three-chamber paradigm test and long-term SRM retention was assessed 7 days after the initial interaction. Mice were bilaterally administered of vehicle (Veh) or CNO into LS 20 min before the initial interaction. Representative images showing the co-expression of hM4D(Gi)-mCherry and STEP immunoreactivity in dCA2 pyramidal neurons. Scale bars represent $50 \mathrm{~mm}$. mCherry signals of axonal projections were observed in dLS. Scale bar represents $100 \mathrm{~mm}$. NeuN, neuronal nuclear protein. B Top, schematic representation of the three-chamber sociability test. Bottom left, time spent by the subject mouse in sniffing directed at the wire cage containing the juvenile stimulus mouse or the empty wire cage. Both $\mathrm{hM} 4 \mathrm{D}(\mathrm{Gi}) /$ Veh and $\mathrm{hM} 4 \mathrm{D}(\mathrm{Gi}) / \mathrm{CNO}$ subject mice spent significantly more time interacting with the wire cage containing the juvenile stimulus mouse than the empty wire cage. Bottom right, discrimination index (stimulus minus empty) was similar between $\mathrm{hM} 4 \mathrm{D}(\mathrm{Gi}) / \mathrm{Veh}$ and hM4D(Gi)/CNO subject mice in the sociability test. C Top, schematic representation of the three-chamber social novelty preference test. Bottom left, time spent by the subject mouse in sniffing directed at the wire cage containing a familiar mouse or a novel 1 mouse, 10 min after the sociability test. Both $\mathrm{hM} 4 \mathrm{D}(\mathrm{Gi}) /$ Veh and $\mathrm{hM} 4 \mathrm{D}(\mathrm{Gi}) / \mathrm{CNO}$ subject mice spent significantly more time sniffing the cage containing the novel mouse than the familiar mouse. Bottom right, discrimination index (novel 1 minus familiar) was comparable between $\mathrm{hM} 4 \mathrm{D}(\mathrm{Gi}) /$ Veh and $\mathrm{hM} 4 \mathrm{D}(\mathrm{Gi}) / \mathrm{CNO}$ subject mice in the social novelty preference test. D Top, schematic representation of the three-chamber long-term SRM test. Bottom, time spent by the subject mouse in sniffing directed at the wire cage containing a familiar mouse or a novel 2 mouse, 7 days after the initial interaction. Both hM4D(Gi)/Veh and hM4D(Gi)/CNO subject mice spent significantly more time sniffing the cage containing the novel mouse than the familiar mouse. Bottom right, discrimination index (novel 2 minus familiar) was similar between hM4D(Gi)/Veh and 
$\mathrm{hM} 4 \mathrm{D}(\mathrm{Gi}) / \mathrm{CNO}$ subject mice in 7-day long-term SRM test. The total number of animal examined is indicated by $n$ in parenthesis. Error bars represent the SEM; ${ }^{*} p<0.05,{ }^{\star *} p<0.01$, ${ }^{\star \star *} p<0.001$.

\section{Figure 8}

Activation of dCA2-to-vCA1 projections rescues long-term SRM deficit in Oxtr ${ }^{-/-}$mice. A Schematic representation of viral injection and CNO administration. Three weeks after stereotaxic injection of $\mathrm{AAV}_{5^{-}}$ hSyn-DIO-hM3D(Gq)-mCherry or AAV ${ }_{5}$-hSyn-DIO-mCherry into the dCA2 of Oxtr ${ }^{-1}$ mice, mice were subjected to three-chamber paradigm test and long-term SRM retention was tested 7 days after the initial interaction. $\mathrm{Oxtr}^{-1-}$ mice were bilaterally administered of CNO into vCA1 $20 \mathrm{~min}$ before the initial interaction. Representative images showing the co-expression of hM3D(Gq)-mCherry and STEP immunoreactivity in dCA2 pyramidal neurons of $\mathrm{Oxtr}^{-1-}$ mice. Scale bar represents $50 \mathrm{~mm}$. mCherry signals of axonal projections were observed in vCA1. Scale bar represents $100 \mathrm{~mm}$. NeuN, neuronal nuclear protein; SO, stratum oriens; SP, stratum pyramidale; SR, stratum radiatum. B Top, schematic representation of the three-chamber sociability test. Bottom left, time spent by the subject mouse in sniffing directed at the wire cage containing the juvenile stimulus mouse or the empty wire cage. Both $\mathrm{mCherry} / \mathrm{CNO}$ and $\mathrm{hM} 3 \mathrm{D}(\mathrm{Gq}) / \mathrm{CNO} O \mathrm{xtr}^{2 /-}$ mice spent significantly more time interacting with the wire cage containing the juvenile stimulus mouse than the empty wire cage. Bottom right, discrimination index (stimulus minus empty) were similar between mCherry/CNO and hM3D(Gq)/CNO Oxtr/- mice in the sociability test. C Top, schematic representation of the three-chamber social novelty preference test. Bottom left, time spent by the subject mouse in sniffing directed at the wire cage containing a familiar mouse or a novel 1 mouse, 10 min after the sociability test. Both mCherry/CNO and $\mathrm{hM} 3 \mathrm{D}(\mathrm{Gq}) / \mathrm{CNO}$ Oxtr/- mice spent significantly more time sniffing the cage containing the novel mouse than the familiar mouse. Bottom right, discrimination index (novel 1 minus familiar) was comparable between mCherry/CNO and $\mathrm{hM} 3 \mathrm{D}(\mathrm{Gq}) / \mathrm{CNO}$ Oxtr ${ }^{-/}$mice in the social novelty preference test. D Top, schematic representation of the three-chamber long-term SRM test. Bottom, time spent by the subject mouse in sniffing directed at the wire cage containing a familiar mouse or a novel 2 mouse, 7 days after the initial interaction. $\mathrm{hM} 3 \mathrm{D}(\mathrm{Gq}) / \mathrm{CNO}$, but not mCherry/CNO, Oxtr/- mice spent significantly more time sniffing the cage containing the novel mouse than the familiar mouse. Bottom right, discrimination index (novel 2 minus familiar) of hM3D(Gq)/CNO Oxtr/- mice was significantly higher than mCherry/CNO Oxtr ${ }^{-/}$ mice in 7-day long-term SRM test. The total number of animal examined is indicated by $n$ in parenthesis. Error bars represent the SEM; ${ }^{*} p<0.05,{ }^{* \star} p<0.01,{ }^{\star \star \star} p<0.001$. 